\title{
Classificação dos tumores hematopoéticos e linfoides de acordo com a OMS: padronização da nomenclatura em lingua portuguesa, $4^{\mathrm{a}}$ edição
}

\author{
Classification of haematopoietic and lymphoid tumors. WHO, standardization of nomenclature in Portuguese, \\ $4^{\text {th }}$ edition
}

\begin{tabular}{|c|c|}
\hline & 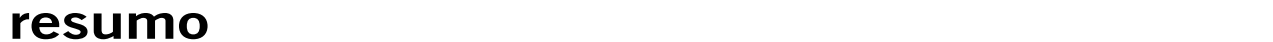 \\
\hline $\begin{array}{l}\text { Organização Mundial da } \\
\text { Saúde }\end{array}$ & trodução: A classificação da Organização Mundial da Saúde (OMS) para os tumores do tecido \\
\hline 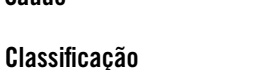 & $\begin{array}{l}\text { hematopoético e linfoide }\left(4^{a} \text { edição, } 2008\right) \text { representa uma revisão atualização da } 3^{a} \text { ediçãao publicada } \\
\text { em 2001. A tradução da nomenclatura utilizada para identificar as entidades descritas deve ser clara, }\end{array}$ \\
\hline 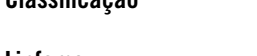 & precisa e uniforme no sentido de reproduzir de forma correta as diversas entidades clinicopatológicas \\
\hline Linfoma & ara clínicos, patologistas e pesquisadores envolvidos na área da onco-hematopatologia. Objetivo: Os \\
\hline Leucemia & $\begin{array}{l}\text { autores apresentam uma proposta de atualização e padronização terminológica em língua portuguesa, } \\
\text { com base na OMS } 2008 \text {. }\end{array}$ \\
\hline
\end{tabular}

abstract

Introduction: The World Health Organization (WHO) classification of hematopoietic and lymphoid tissue (4 $4^{\text {th }}$ edition, 2008) tumors constitutes an updated review of the $3^{\text {rd }}$ edition published in 2001. The translation of the nomenclature used to describe the entities should be clear, precise and uniform so that clinicians, pathologists and researchers involved in the onco-hematopathological area may identify them accurately. Objective: With this purpose, the authors present an updated proposal and a terminological standardization in Portuguese based on WHO/2008.

\begin{abstract}
Maria Cláudia Nogueira Zerbini'; Fernando Augusto Soares ${ }^{2}$; José Carlos Morais ${ }^{3}$; José Vassallo4; Elvira Deolinda Rodrigues Pereira Velloso5; Maria de Lourdes L. F. Chaufaille; Carlos Sergio Chiattone; ${ }^{7}$ Vera Lucia Aldred ${ }^{8}$; Sheila Aparecida Coelho Siqueira9; Antonio Correia Alves ${ }^{10}$;

Jussara Bianchi Castelli ${ }^{11}$; Claudia Regina Gomes Mendes Cardim de Oliveira ${ }^{12}$; Yara de Menezes ${ }^{13}$; Roberto Pinto Paes ${ }^{14}$
\end{abstract}

com base na OMS/2008.

\footnotetext{
1. Doutora em Patologia; professora do Departamento de Patologia da Faculdade de Medicina da Universidade de São Paulo (FMUSP).

2. Professor titular do Departamento de Patologia da Faculdade de Odontologia da USP; diretor do Departamento de Anatomia Patológica do Hospital do Câncer A. C. Camargo.

3. Professor titular do Departamento de Patologia da Universidade Federal do Rio de Janeiro (UFR)).

4. Professor titular do Departamento de Anatomia Patológica da Faculdade de Ciências Médicas da Universidade Estadual de Campinas (FCM/UNICAMP).

5. Doutora em Hematologia; médica hematologista do Hospital das Clínicas (HC) da FMUSP; médica do Laboratório Clínico do Setor de Citogenética do Hospital Israelita Albert Einstein.

6. Professora livre-docente da disciplina de Hematologia da Universidade Federal de São Paulo (UNIFESP); médica do Fleury Medicina Diagnóstica; responsável pelo Laboratório de Citogenética do Fleury Medicina Diagnóstica.

7. Professor adjunto da Faculdade de Ciências Médicas da Santa Casa de São Paulo (FCMSCSP); chefe da disciplina de Hematologia e Oncologia da FCMSCSP.

8. Doutora em Ciências; médica patologista da Divisão de Anatomia Patológica do HC/FMUSP.

9. Doutora em Ciências; médica supervisora do Laboratório de Imuno-histoquímica da Divisão de Anatomia Patológica do HC/FMUSP; professora colaboradora do Departamento de Patologia da FMUSP.

10. Doutor em Patologia; professor do Departamento de Patologia da UNIFESP.

11. Doutora em Ciências; médica patologista do Instituto do Coração da FMUSP.

12. Doutora em Ciências; chefe do Departamento de Patologia do Instituto de Ortopedia e Traumatologia da FMUSP; médica patologista do Fleury Medicina Diagnóstica.

13. Doutora em Patologia; médica patologista do Instituto Adolfo Lutz; médica patologista do Hospital Alemão Oswaldo Cruz (HAOC).

14. Professor adjunto do Departamento de Patologia da FCMSCSP.
} 


\section{Introdução}

A classificação da Organização Mundial da Saúde (OMS) para os tumores do tecido hematopoético e linfoide (4a edição, 2008)(4) representa uma revisão atualizada da $3^{a}$ edição, publicada em $2001^{(2)}$, que tem o mérito de ser a primeira classificação de consenso desse tipo de neoplasias malignas. Os objetivos da nova classificação incluem revisão de critérios, nomenclatura e definições das doenças descritas na $3^{\text {a }}$ edição, assim como inclusão de novas entidades, algumas definitivas e outras provisórias, por não terem definição suficientemente clara nesse momento.

O princípio da classificação da OMS se mantém desde a classificação Revised European-American Classification of Lymphoid Neoplasms (REAL) de 1994(1), representando uma lista de neoplasias definidas por um conjunto de parâmetros clínicos, morfológicos, imunofenotípicos e genéticos, variando a importância de acordo com cada entidade, sem que nenhum seja considerado gold standard.

A tradução da nomenclatura das entidades descritas na classificação da OMS se reveste de importância para que seja utilizada por patologistas, hematologistas e oncologistas de forma padronizada na língua portuguesa. Isso se tornou mais relevante à medida que a classificação foi se consolidando universalmente, passando a ser reconhecida como uma linguagem comum entre todos os especialistas relacionados com essa área da medicina. Uma proposta de padronização terminológica em língua portuguesa para a $3^{a}$ edição foi publicada em $2002^{(3)}$.

Apresentamos a seguir uma proposta de atualização da padronização terminológica em língua portuguesa com base na nova edição da classificação da $\mathrm{OMS} / 2008^{(4)}$. As novas entidades estão apresentadas em negrito e aquelas introduzidas em caráter provisório, em itálico. As siglas para algumas entidades estão entre parênteses. A Classificação Internacional de Doenças - Oncologia (CID-O) é apresentada entre colchetes e em itálico, quando considerado código provisório, a ser incorporado ou modificado na próxima edição da classificação. As entidades relacionadas com a classificação anterior, mas que foram renomeadas, não são consideradas novas entidades.

\section{Neoplasias mieloproliferativas}

- Leucemia mieloide crônica BCR-ABL1 positiva (LMC/BCR-ABL1+) [9875/3]

- Leucemia neutrofílica crônica (LNC) [9963/3]

- Policitemia vera (PV) [9950/3]
- Mielofibrose primária (MFP) [9961/3]

- Trombocitemia essencial (TE) [9962/3]

- Leucemia eosinofílica crônica, sem outras especificações (LEC, SOE) [9964/3]

- Mastocitose

$\sqrt{ }$ Mastocitose cutânea (MC) [9740-1]

$\sqrt{ }$ Mastocitose sistêmica indolente (MSI) [9741/1]

$\sqrt{ }$ Mastocitose sistêmica agressiva (MSA) [9741/3]

$\sqrt{ }$ Mastocitose sistêmica associada à doença clonal hematológica de linhagem não mastocitária (MS-DCHNM) [9741/3]

$\sqrt{ }$ Leucemia de mastócitos (LM) [9742/3]

$\sqrt{ }$ Sarcoma de mastócitos (SMA) [9740/3]

$\sqrt{ }$ Mastocitoma extracutâneo (MEC) [9740/1]

- Neoplasia mieloproliferativa inclassificável (NMI) [9975/3]

II. Neoplasias mieloides e linfoides com eosinofilia e anormalidades dos genes PDGFRA, PDGFRB ou FGFR 1

- Neoplasias mieloides e linfoides com rearranjo do gene PDGFRA [9965/3]

- Neoplasias mieloides com rearranjo do gene PDGFRB [9966/3]

- Neoplasias mieloides e linfoides com anomalias do gene FDFGR1 [9967/3]

\section{Neoplasias mieloproliferativas/ mielodisplásicas (NMP/MD)}

- Leucemia mielomonocítica crônica (LMMC) [9945/3]

- Leucemia mieloide crônica atípica BCR-ABL1 negativa (LMC, BCR/ABL1 neg) [9876/3]

- Leucemia mielomonocítica crônica juvenil (LMMCJ) [9946/3]

- Neoplasia mieloproliferativa/mielodisplásica inclassificável (NMP/MD-I) [9975/3]

- Anemia refratária com sideroblastos em anel associada à intensa trombocitose (ARSA/T) [9982/3] 


\section{Síndromes mielodisplásicas (SMD)}

- Citopenia refratária com displasia (de uma linhagem ou unilinear) (CR)

$\sqrt{ }$ Anemia refratária (SMD/CR-AR) [9980/3]

$\sqrt{ }$ Neutropenia refratária (SMD/CR-NR) [9991/3]

$\sqrt{ }$ Trombocitopenia refratária (SMD/CR-TR) [9992/3]

- Anemia refratária com sideroblastos em anel (SMD/ ARSA) [9982/3]

- Citopenia refratária com displasia (de múltiplas linhagens ou multilinear) (SMD/CR-DML) [9985/3]

- Anemia refratária com excesso de blastos (SMD/ AREB) [9983/3]

- Síndrome mielodisplásica associada a del (5q) isolada (SMD/5q-) [9986-3]

- Síndrome mielodisplásica, inclassificável (SMD/I) [9989/3]

- Síndrome mielodisplásica pediátrica (SMD-P)

$\sqrt{ }$ Citopenia refratária pediátrica (SMD-P/CR) [9985-3]

\section{Leucemia mieloide aguda (LMA) e neoplasias de células precursoras relacionadas}

- LMA com anormalidades genéticas recorrentes

$\sqrt{ }$ LMA com t(8;21)(q22; q22); RUNX1-RUNX1T1 [9896/3]

$\sqrt{ }$ LMA com inv(16)(p13.1;q22); CBFB-MYH11 [9871/3]

$\sqrt{ }$ Leucemia promielocítica aguda (LPMA) com $\mathrm{t}(15 ; 17)(\mathrm{q} 22 ; \mathrm{q} 12) ;$ PML-RARA [9866/3]

$\sqrt{ }$ LMA com $\mathrm{t}(9 ; 11)(\mathrm{p} 22 ; \mathrm{q} 23) ;$ MLLT3-MLL [9897/3]

$\sqrt{ }$ LMA com $t(6 ; 9)(p 23 ; q 34) ;$ DEK-NUP214 [9865/3]

$\sqrt{ }$ LMA com inv(3)(q21;q26.2) ou $\mathrm{t}(3 ; 3)$ (q21;q26.2); RPN1-EVI1 [9869/3]

$\sqrt{ }$ LMA (megacarioblástica) com $t(1 ; 22)$ (p13;q13); RBM15-MKL1 [9911/3]

$\sqrt{ }$ LMA com mutação de NPM1 [9861/3]

$\sqrt{ }$ LMA com mutação de CEBPA [9861/3]
- LMA com alterações relacionadas com mielodisplasia (LMA/MD) [9895/3]

- Neoplasias mieloides relacionadas com terapia (NM-T) [9920/3]

- Leucemia mieloide aguda, SOE (LMA, SOE) [9861/3]

$\sqrt{ }$ LMA com diferenciação mínima [9872/3]

$\sqrt{ }$ LMA sem maturação [9873/3]

$\sqrt{ }$ LMA com maturação [9874/3]

$\sqrt{ }$ Leucemia mielomonocítica aguda (LMMA) [9867/3]

$\sqrt{ }$ Leucemia monoblástica (LMoB) e leucemia monocítica (LMoC) [9891/3]

$\sqrt{ }$ Leucemia eritroide aguda (LEA) [9840/3]

$\sqrt{ }$ Leucemia megacarioblástica aguda (LMegA) [9910/3]

$\sqrt{ }$ Leucemia basofílica aguda (LBA) [9870/3]

$\sqrt{ }$ Panmielose aguda com mielofibrose (PMA-MF) [9931/3]

- Sarcoma mieloide (SM) [9930/3]

- Proliferações mieloides relacionadas com síndrome de Down

$\sqrt{ }$ Mielopoese anormal transitória (D-MAT) [9898/1]

$\sqrt{ }$ Leucemia mieloide associada à síndrome de Down (D-LMA) [9898/3]

- Neoplasia da célula dendrítica plasmocitoide blástica (NCDPB) [9727/3]

\section{Leucemias agudas de linhagem ambígua}

- Leucemia aguda indiferenciada (LAI) [9801/3]

- Leucemia aguda de fenótipo misto (LAFM) com $\mathrm{t}(9 ; 22)(q 34 ; q 11.2) ;$ BCR-ABL1 [9806/3]

- Leucemia aguda de fenótipo misto (LAFM) com $t(v ; 11 q 23) ;$ MLL rearranjado [9807/3]

- Leucemia aguda de fenótipo misto (LAFM) B/mieloide, SOE [9808/3]

- Leucemia aguda de fenótipo misto (LAFM) $\mathrm{T} /$ mieloide, SOE [9808/3]

- Leucemia/linfoma linfoblástico de células NK (LLL-NK) 


\section{Neoplasias de células linfoides precursoras}

- Leucemia/linfoma linfoblástico B (LLL-B)

$\sqrt{ }$ Leucemia/linfoma linfoblástico B, SOE [9811/3]

$\sqrt{ }$ Leucemia/linfoma linfoblástico $B$, com anormalidades genéticas recorrentes

$\checkmark$ Leucemia/linfoma linfoblástico $B$ com $\mathrm{t}(9 ; 22)(\mathrm{q} 34 ; \mathrm{q} 11.2) ; B C R-A B L 1$ [9812/3]

$\checkmark$ Leucemia/linfoma linfoblástico $B$ com $\mathrm{t}(\mathrm{v} ; 11 \mathrm{q} 23) ; M L L$ rearranjado [9813/3]

$\checkmark$ Leucemia/linfoma linfoblástico B com $\mathrm{t}(12 ; 21)(\mathrm{p} 13 ; \mathrm{q} 22) ; T E L-A M L 1$ (ETV6$-R U N X 1)[9814 / 3]$

$\checkmark$ Leucemia/linfoma linfoblástico B com hiperdiploidia [9815/3]

$\checkmark$ Leucemia/linfoma linfoblástico B com hipodiploidia [9816/3]

$\checkmark$ Leucemia/linfoma linfoblástico B com $\mathrm{t}(5 ; 14)(\mathrm{q} 31 ; \mathrm{q} 32) ; \mathrm{IL} 3 / \mathrm{IGH}[9817 / 3]$

$\checkmark$ Leucemia/linfoma linfoblástico $B$ com $\mathrm{t}(1 ; 19)(\mathrm{q} 23 ; \mathrm{p} 13.3) ;$ E2A-PBX1 (TCF3-PBX1) [9813/3]

- Leucemia/linfoma linfoblástico T (LLL-T) [9837/3]

\section{Neoplasias de células linfoides B maduras}

- Leucemia linfoide crônica/linfoma linfocítico (LLC/LL) [9823/3]

- Leucemia prolinfocítica B (LP-B) [9833/3]

- Linfoma B da zona marginal esplênica (LZME)[9689/3]

- Tricoleucemia (TRL) [9940/3]

- Linfoma/leucemia esplênico de células B, inclassificável [9591/3]

$\sqrt{ }$ Linfoma esplênico difuso da polpa vermelha, de linfócitos B pequenos [9591/3]

$\sqrt{ }$ Tricoleucemia variante (TRL-V) [9591/3]

- Linfoma linfoplasmocítico (LLPL) [9671/3]

$\sqrt{ }$ Macroglobulinemia de Waldeström (MW) [9761/3]

- Doenças da cadeia pesada (DCP) [9762/3]

$\sqrt{ }$ Doença da cadeia pesada alfa [9762/3]

$\sqrt{ }$ Doença da cadeia pesada gama [9762/3]

$\sqrt{ }$ Doença da cadeia pesada mu [9762/3]
- Mieloma múltiplo (MM) [9732/3]

- Plasmocitoma solitário do osso [9731/3]

- Plasmocitoma extraósseo [9734/3]

- Linfoma da zona marginal extranodal do tecido linfoide associado à mucosa (Linfoma MALT) [8688/3]

- Linfoma da zona marginal nodal (LZMN) [9699/3]

$\sqrt{ }$ Linfoma da zona marginal nodal pediátrico (LZMN-P) [9699/3]

- Linfoma folicular (LF) [9690/3]

$\sqrt{ }$ Linfoma folicular pediátrico (LF-P) [9690/3]

- Linfoma centrofolicular primário da pele (LF-Pele) [9597/3]

- Linfoma de células do manto (LCM) [9673/3]

- Linfoma difuso de grandes células B, SOE (LDGCB, SOE) [9680/3]

$\sqrt{ }$ Linfoma de grandes células $B$ rico em células T e histiócitos (LGCB-RTH) [9688/3]

$\sqrt{ }$ Linfoma difuso de grandes células $B$ do sistema nervoso central (LDGCB-SNC) [9680/3]

$\sqrt{ }$ Linfoma difuso de grandes células $B$ da pele, tipo "da perna" (LDGCB-perna) [9680/3]

$\sqrt{ }$ Linfoma difuso de grandes células $B E B V$-positivo do idoso (LDGCB-EBV + idoso) [9680/3]

- Linfoma difuso de grandes células $B$ associado à inflamação crônica (LDGCB-IC) [9680/3]

- Granulomatose linfomatoide (GL) [9766/1]

- Linfoma de grandes células B do mediastino (tímico) (LGCB-Med) [9679/3]

- Linfoma de grandes células B intravascular (LGCB-IV) [9712/3]

- Linfoma de grandes células B ALK-positivo (LGCB-ALK+) [9737/3]

- Linfoma plasmoblástico (LPb) [9735/3]

- Linfoma de grandes células B com origem na D. de Castleman multicêntrica associada ao HHV-8 (LGCB/DCM-HHV8+) [9738/3]

- Linfoma primário de efusões (LPE) [9678/3]

- Linfoma de Burkitt (LB) [9687/3]

- Linfoma de células B inclassificável, com características intermediárias entre o $L D G C B$ e o Linfoma de Burkitt (LBI-LDGCB/LB) [9680/3]

- Linfoma de células B inclassificável, com características intermediárias entre o $L D G C B$ e o Linfoma de Hodgkin (LBI-LDGCB/LH) [9596/3] 


\section{Neoplasias de}

\section{células T e NK maduras}

- Leucemia prolinfocítica de células T (LPL-T) [9834/3]

- Leucemia linfocítica de células T granulares grandes (LLTGG) [9831/3]

$\sqrt{ }$ Doença linfoproliferativa crônica de células NK (DLPC-NK) [9831/3]

- Leucemia agressiva de células NK (LA-NK) [9948/3]

- Doença linfoproliferativa sistêmica de células T EBV+ da pediátrica (DLPST-EBV+I) [9724/3]

- Linfoma Hydroa vacciniforme símile (LHV) [9725/3]

- Leucemia/linfoma de células T do adulto (LLTA) [9827/3]

- Linfoma de células NK/T, tipo nasal (LNK/T-nasal) [9719/3]

- Linfoma de células T associado à enteropatia (LT-E) [9717/3]

- Linfoma de células T hepatoesplênico (LT-HE) [9716/3]

- Linfoma de células T subcutâneo paniculite-símile (LT-SPS) [9708/3]

- Micose fungoide (MF) [9700/3]

- Síndrome de Sézary (SS) [9701/3]

- Doenças linfoproliferativas de células T CD30 positivas primárias da pele

$\sqrt{ }$ Papulose linfomatoide (PL) [9718/1]

$\sqrt{ }$ Linfoma de grandes células anaplásicas primário da pele (LGCA-pele) [9718/3]

- Linfoma de células T gama-delta primário da pele (LTGD-pele) [9726/3]

- Linfoma agressivo de células T citotóxicas CD8 positivas, epidermotrópico primário da pele [9709/3]

- Linfoma de células T pequenas/médias CD4 positivas, primário da pele [9709/3]

- Linfoma de células T periféricas, SOE (LTP, SOE) [9702/3]

- Linfoma de células T angioimunoblástico (LTAI) [9705/3]

- Linfoma de grandes células anaplásicas, ALK positivo (LGCA-ALK+) [9714/3]
- Linfoma de grandes células anaplásicas, ALK negativo (LGCA-ALK-) [9702/3]

\section{Linfoma de Hodgkin (LH)}

- Linfoma de Hodgkin, predominância linfocítica nodular (LH-PLN) [9659/3]

- Linfoma de Hodgkin clássico (LH-C) [9650/3]

$\sqrt{ }$ Linfoma de Hodgkin clássico, esclerose nodular (LHC-EN) [9663/3]

$\sqrt{ }$ Linfoma de Hodgkin clássico, rico em linfócitos (LHC-RL) [9651/3]

$\sqrt{ }$ Linfoma de Hodgkin clássico, celularidade mista (LHC-CM) [9652/3]

$\sqrt{ }$ Linfoma de Hodgkin clássico, depleção linfocitária (LHC-DL) [9653/3]

\section{Neoplasia de células histiocíticas e dendríticas}

- Sarcoma histiocítico (SH) [9755/3]

- Histiocitose de células de Langerhans (HCLa) [9751/3]

- Sarcoma de células de Langerhans (SCL) [9756/3]

- Sarcoma de células dendríticas interdigitantes (SCDI) [9757/3]

- Sarcoma de células dendríticas foliculares (SCDF) [9758/3]

- Tumor de células reticulares fibroblásticas (TCRF) [9759/3]

- Tumor de células dendríticas indeterminadas (TCDI) [9757/3]

- Xantogranuloma juvenil disseminado (XGJD) [9757/3]

\section{Doenças linfoproliferativas} associadas à imunodeficiência (DLP-ID)

- Doenças linfoproliferativas associadas à imunodeficiência primária (DLP-IDP)

- Doenças linfoproliferativas associadas à infecção pelo HIV (DLP-HIV)

- Doenças linfoproliferativas pós-transplante (DLP-PT) $\sqrt{ }$ Lesões iniciais 
$\checkmark \quad$ Hiperplasia plasmocítica [9971/1]

$\checkmark$ DLPT monucleose símile [9971/1]

$\diamond$ DLPT polimórfica [9971/3]

$\diamond$ DLPT monomórfica (tipos $\mathrm{B}, \mathrm{T}$ e NK)*

$\diamond \quad$ DLPT tipo linfoma de Hodgkin clássico*
- Outras doenças linfoproliferativas associadas à imunodeficiência iatrogênica (DLP- IDI)

*Essas lesões são classificadas de acordo com a leucemia ou o linfoma correspondente e recebem o código respectivo.

\section{Referências}

1. HARRIS, N. et al. A revised European-American classification of lymphoid neoplasms: a proposal of the International Lymphoma Study Group. Blood, v. 84, p. 1361-92, 1994.

2. JAFFE, E. S. et al. (Ed.). Tumors of haematopoietic and lymphoid tissues. Lyon: IARC Press, 2001.

3. PAES, R. A. P. et al. Classificação da Organização Mundial da Saúde para as neoplasias dos tecidos hematopoético e linfoide: proposta de padronização terminológica em língua portuguesa do Grupo de Hematopatologia da Sociedade Brasileira de Patologia. Rio de Janeiro, 2002. v. 38, p. 237-9.

4. SWERDLOW, S. H. et al. (Ed.). World Health Organization classification of tumors of haematopoietic and lymphoid tissues. Lyon: IARC, 2008. 